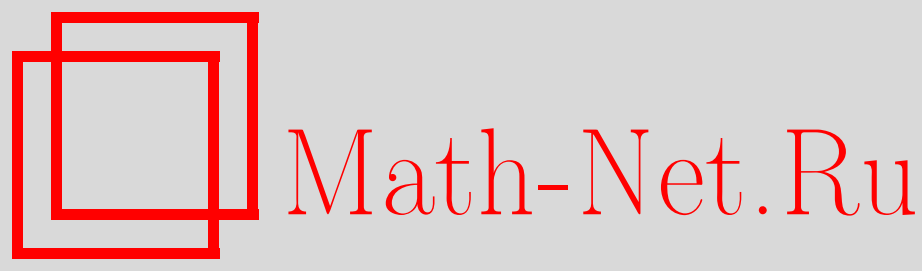

М. Ю. Ковалевский, Квазисредние в решении задачи классификации состояний равновесия конденсированных сред со спонтанно нарушенной симметрией, $Т M \Phi$, 2009, том 160, номер 2, 290-303

DOI: https://doi.org/10.4213/tmf6399

Использование Общероссийского математического портала Math-Net.Ru подразумевает, что вы прочитали и согласны с пользовательским соглашением http://www.mathnet.ru/rus/agreement

Параметры загрузки:

IP: 35.173 .219 .149

26 апреля 2023 г., 15:07:39

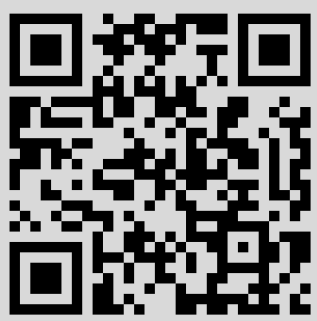




\title{
КВАЗИСРЕДНИЕ В РЕШЕНИИ ЗАДАЧИ КЛАССИФИКАЦИИ СОСТОЯНИЙ РАВНОВЕСИЯ КОНДЕНСИРОВАННЫХ СРЕД СО СПОНТАННО НАРУШЕННОЙ СИММЕТРИЕЙ
}

\begin{abstract}
Развит статистический подход к решению задачи классификации состояний равновесия вырожденных конденсированных сред. Введены генераторы ненарушенной и пространственной симметрий состояния равновесия, на основе которых получены уравнения классификации для параметра порядка. Выявлен механизм появления дополнительных термодинамических параметров, характеризующих как однородные, так и неоднородные состояния равновесия. Найдены аналитические решения задачи классификации состояний равновесия для ряда жидких кристаллов.
\end{abstract}

Ключевые слова: равновесие, квазисреднее, параметр порядка, уравнение классификации, жидкий кристалл, термодинамические параметры.

\section{1. МОТИВАЦИЯ ИССЛЕДОВАНИЙ И ПОСТАНОВКА ЗАДАЧИ}

Теоретическим фундаментом статистической физики, описывающей равновесные состояния конденсированных сред со спонтанно нарушенной симметрией, является концепция квазисредних [1], [2]. Ее конструктивный момент - введение в равновесный статистический оператор бесконечно малого источника, который понижает симметрию состояния статистического равновесия по сравнению с симметрией гамильтониана и позволяет обобщить распределение Гиббса на вырожденные конденсированные среды. Развитие концепции квазисредних и ее приложение к квантовым жидкостям со скалярным и тензорным параметрами порядка можно найти в рабо$\operatorname{Tax}[3]-[6]$.

Состояния равновесия со спонтанно нарушенной симметрией, возникающие в результате фазового перехода второго рода в конденсированных средах, можно описать и классифицировать в рамках феноменологической теории [7]. На основе модельного вида свободной энергии проводились исследования для сред с различным

*Национальный научный центр "Харьковский физико-технический институт", Харьков, Украина; Белгородский государственный университет, Белгород, Россия.

E-mail: mik@kipt.kharkov.ua 
видом параметра порядка, в частности для сверхтекучих систем [8] и жидких кристаллов [9]. Соображения симметрии налагают ограничения на явный вид свободной энергии как функционала параметра порядка, однако попытки связать феноменологические параметры разложения с параметрами межчастичного взаимодействия сталкиваются с немалыми трудностями [10]. Другим осложнением указанного подхода является то, что он справедлив только в окрестности точки фазового перехода, где параметр порядка мал. Вдали от критической температуры возникают трудности с выбором явного вида свободной энергии как функционала параметра порядка, а также с решением соответствующих нелинейных уравнений для этого функционала [11]. Теоретико-групповой подход [12], [13] использует представление о ненарушенной симметрии вырожденного состояния равновесия как подгруппе симметрии нормальной фазы. В работах [14]-[16] проводилась классификация состояний в рамках этого подхода для сверхтекучего ${ }^{3} \mathrm{He}$, характеризующегося тензорным параметром порядка.

Уменьшение симметрии состояния равновесия в результате фазового перехода приводит к необходимости обобщения термодинамики конденсированной среды и требует введения дополнительных параметров, адекватно описывающих вырожденные состояния. Установление характера и вида этих параметров представляет собой основную задачу наших исследований. В статье предложен метод классификации состояний равновесия вырожденных конденсированных сред на основе введенных представлений генераторов ненарушенной и пространственной симметрий. Показана роль симметрии гамильтониана и трансформационных свойств параметра порядка относительно глобальных преобразований, генераторами которых являются интегралы движения, для классификации таких состояний равновесия. Решение этой задачи опирается на условия ненарушенной и пространственной симметрий при ненулевых значениях параметра порядка. Нами детально изучены конденсированные среды со спонтанно нарушенной трансляционной и вращательной инвариантностью. Установлена связь условий симметрии с одноосной и двухосной структурами параметра порядка жидких кристаллов с нематическим и холестерическим упорядочением в случае непрерывного характера свойств симметрии состояния равновесия. В случае дискретных свойств симметрии состояния равновесия выяснена связь структуры генераторов ненарушенной и пространственной симметрии со смектическим упорядочением.

\section{2. НОРМАЛЬНОЕ СОСТОЯНИЕ РАВНОВЕСИЯ КОНДЕНСИРОВАННОЙ СРЕДЫ}

Распределение Гиббса для нормальной немагнитной конденсированной среды в лабораторной системе отсчета $\widehat{w}(Y)=e^{\Omega(Y)-Y_{a} \hat{\gamma}_{a}}$ зависит от термодинамических параметров $Y_{a}$, связанных с симметрией гамильтониана. Эти величины включают в себя $Y_{0}^{-1} \equiv T$ - температуру, $-Y_{k} / Y_{0} \equiv v_{k}$ - макроскопическую скорость, $-Y_{4} / Y_{0} \equiv$ $\mu$ - химический потенциал и сопряжены аддитивным интегралам движения $\hat{\gamma}_{a}=$ $\widehat{H}, \widehat{P}_{k}, \widehat{N}$ соответственно. Здесь $\widehat{H}$ - гамильтониан, $\widehat{P}_{k}-$ импульс, $\widehat{N}-$ оператор 
числа частиц. Соотношение симметрии

$$
\left[\widehat{w}, \widehat{P}_{i}\right]=0
$$

отражает трансляционную инвариантность, а формула

$$
[\widehat{w}, \widehat{N}]=0
$$

- фазовую инвариантность состояния равновесия. Равенство

$$
\left[\widehat{w}, \hat{L}_{k}(\mathbf{Y})\right]=0
$$

описывает свойство одноосной анизотропии равновесного статистического оператора при пространственных поворотах; оно выражено в терминах обобщенного оператора орбитального момента $\hat{L}_{i}(\mathbf{Y}) \equiv \hat{L}_{i}+\hat{L}_{i}^{\mathbf{Y}}$, где $\hat{L}_{i}$ - оператор орбитального момента, $\hat{L}_{i}^{\mathbf{Y}} \equiv-\mathrm{i} \varepsilon_{i k l} Y_{k} \partial / \partial Y_{l},\left(Y_{k} /|\mathbf{Y}|\right.$ - ось пространственной анизотропии). Он действует в гильбертовом пространстве и в пространстве термодинамических функций. Лабораторная и собственная системы отсчета связаны преобразованием Галилея, которое определяется унитарным оператором,

$$
U_{\mathbf{u}}=\exp \left\{-\mathrm{i} u_{i} \int d^{3} x x_{i} m \hat{n}(\mathbf{x})\right\},
$$

где $m$ - масса частицы и $u_{i}$ - параметр преобразования. В собственной системе отсчета статистический оператор Гиббса имеет вид

$$
w\left(Y^{\prime}\right)=U_{\mathbf{u}} \widehat{w}(Y) U_{\mathbf{u}}^{+} \equiv e^{\Omega-Y_{a}^{\prime} \hat{\gamma}_{a}},
$$

где

$$
Y_{0}^{\prime}=Y_{0}, \quad Y_{k}^{\prime}=Y_{k}+Y_{0} u_{k}, \quad Y_{4}^{\prime}=Y_{4}+Y_{k} u_{k}+\frac{Y_{0} u^{2}}{2} .
$$

При $u_{k}=-Y_{k} / Y_{0}$ имеем $Y_{k}^{\prime}=0$. Нормальная конденсированная среда в собственной системе отсчета изотропна: $\left[\widehat{w}\left(Y^{\prime}\right), \hat{L}_{k}\right]=0$.

\section{3. КВАЗИСРЕДНИЕ. УРАВНЕНИЯ КЛАССИФИКАЦИИ ПАРАМЕТРА ПОРЯДКА}

В случае фазовых превращений второго рода конденсированная среда из нормального состояния переходит в состояние со спонтанно нарушенной симметрией. Дополнительные термодинамические параметры зависят от характера нарушенной симметрии и связаны с наличием остаточной симметрии состояния равновесия. Выбор параметра порядка связан с конкретной природой равновесных состояний среды. Согласно концепции квазисредних распределение Гиббса для вырожденных сред имеет вид $\widehat{w}_{v} \equiv e^{\Omega_{v}-Y_{a} \hat{\gamma}_{a}-v \widehat{F}}$. Источник $\widehat{F}$, нарушающий симметрию нормального состояния равновесия, представляет собой линейный функционал оператора параметра порядка $\hat{\Delta}_{a}(\mathbf{x})$ :

$$
\widehat{F}=\int d^{3} x\left(f_{a}(\mathbf{x}) \hat{\Delta}_{a}(\mathbf{x})+\text { э.c. }\right) .
$$


В вырожденном состоянии равновесное среднее параметра порядка не равно нулю:

$$
\Delta_{a}(\mathbf{x}, Y, f)=\operatorname{Sp} \widehat{w} \hat{\Delta}_{a}(\mathbf{x}) \equiv \lim _{v \rightarrow 0} \lim _{V \rightarrow \infty} \operatorname{Sp} \widehat{w}_{v} \hat{\Delta}_{a}(\mathbf{x}) \neq 0 .
$$

Эта формула связывает равновесное среднее параметра порядка с термодинамическими силами и функцией $f_{a}(\mathbf{x})$, входящей в источник.

С целью получения уравнений классификации состояний равновесия сформулируем трансформационные свойства оператора параметра порядка. Так как операторы параметра порядка $\hat{\Delta}_{a}(\mathbf{x})$ линейны или билинейны по полевым операторам, правые стороны квантовых скобок Пуассона интегралов движения с операторами параметра порядка будут некоторыми линейными функциями от этих операторов. Условие трансляционной инвариантности имеет вид

$$
\mathrm{i}\left[\widehat{P}_{k}, \hat{\Delta}_{a}(\mathbf{x})\right]=-\nabla_{k} \hat{\Delta}_{a}(\mathbf{x}) .
$$

Генератором группы фазовых преобразований является оператор числа частиц, и оператор параметра порядка $\hat{\Delta}_{a}(\mathbf{x})$ преобразуется согласно соотношению

$$
\left[\widehat{N}, \hat{\Delta}_{a}(\mathbf{x})\right]=-g \hat{\Delta}_{a}(\mathbf{x})
$$

где постоянная величина $g$ зависит от тензорной размерности и внутренней структуры оператора параметра порядка. При пространственных поворотах операторы параметра порядка преобразуются в соответствии с формулой

$$
\mathrm{i}\left[\hat{L}_{l}, \hat{\Delta}_{a}(\mathbf{x})\right]=-g_{l a b} \hat{\Delta}_{b}(\mathbf{x})-\varepsilon_{l j k} x_{k} \nabla_{j} \hat{\Delta}_{a}(\mathbf{x}),
$$

где $g_{l a b}$ - постоянные величины.

Для трансляционно-инвариантных вырожденных состояний равновесия, удовлетворяющих (2.1), условие ненарушенной (остаточной) симметрии состояния равновесия определяется соотношением

$$
[\widehat{w}, \widehat{T}(\xi, \mathbf{Y})]=0
$$

здесь генератор ненарушенной симметрии

$$
\widehat{T}(\xi, \mathbf{Y}) \equiv a_{i} \hat{L}_{i}(\mathbf{Y})+c \widehat{N}
$$

представляет собой линейную комбинацию интегралов движения с действительными параметрами $\mathbf{Y}$ и $\xi \equiv\left(a_{i}, c\right)$ и отражает наличие в вырожденной среде остаточной симметрии, которая меньше чем в более симметричном нормальном состоянии равновесия. Соотношения (2.1), (3.4), (3.5) приводят к дифференциальным уравнениям на функцию $f_{a}(\mathbf{x})$, которые получаются из условия $[\widehat{T}, \widehat{F}]=0$, однако более наглядным является получение уравнений классификации непосредственно для равновесного среднего параметра порядка. Для этого умножим соотношение (3.4) на оператор параметра порядка, учтем трансформационные свойства (3.1)-(3.3) и произведем усреднение. В результате свойство ненарушенной симметрии в случае 
пространственно-однородных состояний равновесия приводит к линейному дифференциальному уравнению с частными производными для параметра порядка:

$$
a_{i}\left(g_{i a b} \Delta_{b}(\mathbf{x})+\varepsilon_{i k l} Y_{k} \frac{\partial \Delta_{a}(\mathbf{x})}{\partial Y_{l}}\right)+\mathrm{i} g c \Delta_{a}(\mathbf{x})=0 .
$$

Условие пространственной симметрии (2.1) влечет равенство $\nabla_{k} \Delta_{a}(\mathbf{x})=0$. Линейность коммутационных соотношений (3.1)-(3.3) для оператора параметра порядка приводит к линейности уравнений для определения его равновесной структуры. В случае $\mathbf{Y}=0$ уравнение (3.6) существенно упрощается и становится линейным однородным алгебраическим уравнением

$$
T_{a b}(\xi, 0) \Delta_{b}=0, \quad T_{a b}(\xi, 0) \equiv a_{i} g_{i a b}+\mathrm{i} g c \delta_{a b} .
$$

Условие нетривиальности решения, $\Delta_{a} \neq 0$, влечет уравнение $\operatorname{det}\left|T_{a b}(\xi, 0)\right|=0$ на допустимые значения параметров генератора ненарушенной симметрии, что и дает возможность провести классификацию состояний равновесия вырожденных конденсированных сред. При этом статистический оператор Гиббса $\widehat{w}=\widehat{w}(Y, \xi)$ зависит как от термодинамических параметров $Y$, так и от параметров $\xi$ генератора ненарушенной симметрии.

Аналогичным образом получим уравнения классификации состояний равновесия в неоднородном случае. Для пространственно-неоднородных состояний вырожденных конденсированных сред генераторы ненарушенной (остаточной) и пространственной симметрий определяются, соответственно, равенствами

$$
\begin{aligned}
\widehat{T}(\xi, \mathbf{Y}) & \equiv a_{i} \hat{L}_{i}(\mathbf{Y})+c \widehat{N}+d_{i} \widehat{P}_{i} \\
\widehat{P}_{k}(\eta, \mathbf{Y}) & \equiv \widehat{P}_{k}-p_{k} \widehat{N}-t_{k j} \hat{L}_{j}(\mathbf{Y})
\end{aligned}
$$

и ведут к соотношениям

$$
\operatorname{Sp}[\widehat{w}, \widehat{T}(\xi, \mathbf{Y})] \hat{\Delta}_{a}(\mathbf{x})=0, \quad \operatorname{Sp}\left[\widehat{w}, \widehat{P}_{k}(\eta, \mathbf{Y})\right] \hat{\Delta}_{a}(\mathbf{x})=0
$$

Здесь $\xi \equiv(\mathbf{a}, c, \mathbf{d})$ и $\eta \equiv\left(\mathbf{p}, t_{k j}\right)$ - действительные параметры, которые характеризуют генераторы ненарушенной и пространственной симметрий. Соотношения (3.9) приводят к зависимости параметра порядка от координаты, а также от параметров ненарушенной и пространственной симметрий. Для того чтобы установить структуру параметра порядка в состоянии равновесия и определить допустимый вид генераторов симметрии, соотношения (3.9) следует дополнить уравнениями

$$
\operatorname{Sp}\left[\widehat{w},\left[\widehat{T}(\xi, \mathbf{Y}), \widehat{P}_{k}(\eta, \mathbf{Y})\right]\right] \hat{\Delta}_{a}(\mathbf{x})=0, \quad \operatorname{Sp}\left[\widehat{w},\left[\widehat{P}_{i}(\eta, \mathbf{Y}), \widehat{P}_{k}(\eta, \mathbf{Y})\right]\right] \hat{\Delta}_{a}(\mathbf{x})=0
$$

которые являются следствиями тождеств Якоби для операторов $\widehat{w}, \widehat{T}(\xi, \mathbf{Y}), \widehat{P}_{k}(\eta, \mathbf{Y})$ и $\widehat{w}, \widehat{P}_{i}(\eta, \mathbf{Y}), \widehat{P}_{k}(\eta, \mathbf{Y})$ соответственно. Условия (3.9), (3.10) приводят к связям между параметрами генераторов симметрии и позволяют решить задачу классификации состояний равновесия неоднородных конденсированных сред. В силу этого 
статистический оператор Гиббса зависит от термодинамических параметров: $\widehat{w}=$ $\widehat{w}(Y, \xi, \eta)$. Учитывая алгебру (3.1)-(3.3), при $\mathbf{Y}=0$ получаем систему уравнений

$$
\begin{gathered}
\nabla_{k} \Delta_{a}(\mathbf{x})=G_{a b}^{k} \Delta_{b}(\mathbf{x}), \quad T_{a b}(\xi, \eta) \Delta_{b}(\mathbf{x})=0, \quad a_{i} \varepsilon_{i k l} G_{a b}^{l} \Delta_{b}(\mathbf{x})=0, \\
a_{i} t_{k j} \varepsilon_{i j \lambda} \varepsilon_{\lambda u v} G_{a b}^{v} \Delta_{b}(\mathbf{x})=0, \quad a_{i} t_{k j} \varepsilon_{i j \lambda} g_{\lambda a b} \Delta_{b}(\mathbf{x})=0, \\
t_{i j} t_{k \lambda} \varepsilon_{j \lambda l} \varepsilon_{l s p} G_{a b}^{p} \Delta_{b}(\mathbf{x})=0, \quad t_{k j} \varepsilon_{j s p} G_{a b}^{k} \Delta_{b}(\mathbf{x})=0, \quad t_{i j} t_{k \lambda} \varepsilon_{j \lambda l} g_{l a b} \Delta_{b}(\mathbf{x})=0,
\end{gathered}
$$

устанавливающую структуру параметра порядка в неоднородном состоянии равновесия. Здесь введены обозначения

$$
G_{a b}^{k} \equiv \mathrm{i} p_{k} g \delta_{a b}+t_{k j} g_{j a b}, \quad T_{a b}(\xi, \eta) \equiv\left(a_{i}+d_{l} t_{l i}\right) g_{i a b}+\mathrm{i} g\left(c+d_{l} p_{l}\right) \delta_{a b} .
$$

Отметим, что уравнения (3.7), (3.11) могут быть полезны также при решении задачи классификации в условиях сосуществования нескольких ненулевых равновесных значений параметров порядка.

\section{4. ЖКИДИЕ КРИСТАЛЛЫ}

Применим сформулированный подход к классификации состояний равновесия жидких кристаллов с нарушенной трансляционной и вращательной симметрией. Оператор параметра порядка для таких сред представляет собой симметричный и бесследовый тензор [9], который мы определим как

$$
\widehat{Q}_{u v}(\mathbf{x}) \equiv \frac{1}{2}\left(\nabla_{u} \hat{\psi}^{+}(\mathbf{x}) \nabla_{v} \hat{\psi}^{+}(\mathbf{x})+\nabla_{v} \hat{\psi}^{+}(\mathbf{x}) \nabla_{u} \hat{\psi}(\mathbf{x})-\frac{2}{3} \delta_{u v} \nabla_{j} \hat{\psi}^{+}(\mathbf{x}) \nabla_{j} \hat{\psi}(\mathbf{x})\right) .
$$

Для этого оператора квантовые скобки с интегралами движения суть

$$
\begin{gathered}
{\left[\widehat{N}, \widehat{Q}_{u v}(\mathbf{x})\right]=0, \quad \mathrm{i}\left[\widehat{P}_{k}, \widehat{Q}_{u v}(\mathbf{x})\right]=-\nabla_{k} \widehat{Q}_{u v}(\mathbf{x}),} \\
\mathrm{i}\left[\hat{L}_{i}, \widehat{Q}_{u v}(\mathbf{x})\right]=-\varepsilon_{i k l} x_{k} \nabla_{l} \widehat{Q}_{u v}(\mathbf{x})-\varepsilon_{i u j} \widehat{Q}_{j v}(\mathbf{x})-\varepsilon_{i v j} \widehat{Q}_{j u}(x) .
\end{gathered}
$$

В силу симметричности и бесследовости параметра порядка его среднее значение $Q_{u v}(\mathbf{x}, \hat{\rho})=\operatorname{Sp} \hat{\rho} \widehat{Q}_{u v}(\mathbf{x})$ содержит пять независимых величин и может быть представлено в виде

$$
Q_{i k}(\mathbf{x}) \equiv Q(\mathbf{x})\left(n_{i}(\mathbf{x}) n_{k}(\mathbf{x})-\frac{\delta_{i k}}{3}\right)+Q^{\prime}(\mathbf{x})\left(m_{i}(\mathbf{x}) m_{k}(\mathbf{x})-\frac{\delta_{i k}}{3}\right),
$$

где $Q, Q^{\prime}$ - модули параметра порядка, $\hat{\rho}$ - произвольный статистический оператор. Взаимно ортогональные единичные векторы $\mathbf{n}$ и $\mathbf{m}$ задают направления анизотропии жидких кристаллов. Так как $m_{u} m_{v}+n_{u} n_{v}+l_{u} l_{v}=\delta_{u v}$, где $\mathbf{m} \times \mathbf{n}=\mathbf{1}$, возможны одноосная или двухосная анизотропия жидкокристаллических состояний равновесия.

В нормальном состоянии конденсированной среды условие пространственной изотропии $\left[\widehat{w}\left(Y^{\prime}\right), \hat{L}_{k}\right]=0$, алгебра квантовых скобок $(4.2)$ и явная формула для среднего значения (4.3) приводят к равенству

$\mathrm{i} \operatorname{Sp}\left[\widehat{w}\left(Y^{\prime}\right), \hat{L}_{i}\right] \widehat{Q}_{u v}(\mathbf{x})=Q\left(Y^{\prime}\right)\left(\varepsilon_{i u j} n_{j} n_{v}+\varepsilon_{i v j} n_{j} n_{u}\right)+Q^{\prime}\left(Y^{\prime}\right)\left(\varepsilon_{i u j} m_{j} m_{v}+\varepsilon_{i v j} m_{j} m_{u}\right)=0$, 
из которого ввиду отсутствия выделенных направлений в этом состоянии равновесия следует, что модули параметра порядка $Q\left(Y^{\prime}\right)=Q^{\prime}\left(Y^{\prime}\right)=0$. Поэтому в системе покоя среднее значение параметра порядка жидких кристаллов обращается в нуль: $\operatorname{Sp} \widehat{w}\left(Y^{\prime}\right) \widehat{Q}_{u v}(\mathbf{x})=0$. В лабораторной системе отсчета, где термодинамический параметр $\mathbf{Y} \neq 0$, параметр порядка отличен от нуля. Из определения (4.1) и соотношений $U_{\mathbf{u}} \hat{\psi}^{+}(\mathbf{x}) U_{\mathbf{u}}^{+}=e^{\mathrm{i} m \mathbf{u x}} \hat{\psi}^{+}(\mathbf{x}), U_{\mathbf{u}} \hat{\psi}(\mathbf{x}) U_{\mathbf{u}}^{+}=e^{\mathrm{i} m \mathbf{u x}} \hat{\psi}(\mathbf{x})$ получим трансформационное свойство параметра порядка при преобразовании Галилея:

$U_{\mathbf{u}} \widehat{Q}_{i k}(\mathbf{x}) U_{\mathbf{u}}^{+}=\widehat{Q}_{i k}(\mathbf{x})+m\left(u_{i} \hat{\pi}_{k}(\mathbf{x})+u_{k} \hat{\pi}_{i}(\mathbf{x})-2 u_{j} \hat{\pi}_{j}(\mathbf{x}) \frac{\delta_{i k}}{3}\right)+m^{2} \hat{n}(\mathbf{x})\left(u_{i} u_{k}-\frac{u^{2} \delta_{i k}}{3}\right)$

(здесь $\hat{\pi}_{k}(\mathbf{x})$ - оператор плотности импульса.) Замечая далее, что в собственной системе отсчета справедливы соотношения $\operatorname{Sp} w\left(Y^{\prime}\right) \hat{\pi}_{k}(\mathbf{x})=0, \operatorname{Sp} w\left(Y^{\prime}\right) \widehat{Q}_{i k}(\mathbf{x})=0$, получим выражение для равновесного значения параметра порядка в лабораторной системе отсчета:

$$
\operatorname{Sp} \widehat{w}(Y) \widehat{Q}_{i k}(\mathbf{x})=m^{2} \operatorname{Sp} \widehat{w} \hat{n}(0) \cdot\left(u_{i} u_{k}-\frac{\delta_{i k} u^{2}}{3}\right) \neq 0 .
$$

Одноосные нематики. Рассмотрим трансляционно-инвариантные состояния равновесия жидкокристаллических сред, удовлетворяющих (2.1), и установим возможные равновесные структуры параметра порядка. Анализ трансляционно-инвариантных подгрупп ненарушенной симметрии равновесных состояний осуществим в собственной системе отсчета исходя из соотношения

$$
\left[\widehat{w}\left(Y^{\prime}\right), \widehat{T}\right]=0 \text {. }
$$

Генератор ненарушенной симметрии $\widehat{T}$ представляет собой линейную комбинацию интегралов движения. Учитывая, что фазовая инвариантность в жидких кристаллах не нарушена, $\left[\widehat{w}\left(Y^{\prime}\right), \widehat{N}\right]=0$, имеем

$$
\widehat{T} \equiv a_{i} \hat{L}_{i}
$$

где $a_{i}$ - действительный вектор, характеризующий повороты в пространстве. Не ограничивая общности рассмотрения, полагаем, что вектор а единичный: $a^{2}=1$. Унитарные преобразования $U=e^{\mathrm{i} \widehat{T}(\mathbf{a})}$ образуют непрерывные подгруппы ненарушенной симметрии, $U(\mathbf{a}) U\left(\mathbf{a}^{\prime}\right)=U\left(\mathbf{a}^{\prime \prime}\right), \mathbf{a}^{\prime}=\mathbf{a}^{\prime \prime}\left(\mathbf{a}, \mathbf{a}^{\prime}\right)$, равновесного состояния. Согласно (4.6), (4.7) имеем $\operatorname{Sp}\left[\widehat{w}\left(Y^{\prime}\right), a_{i} \hat{L}_{i}\right] \widehat{Q}_{u v}(\mathbf{x})=0$. Поэтому, учитывая алгебру (4.2), приходим к уравнению

$$
F_{j k}^{u v} Q_{j k}=0, \quad F_{j k}^{u v} \equiv a_{i}\left(\varepsilon_{i u j} \delta_{v k}+\varepsilon_{i u v} \delta_{u k}\right) .
$$

Перейдем в этой формуле от двойного суммирования к одинарному, заменяя две пары индексов $u, v$ и $j, k$, каждый из которых принимает значения $1,2,3$, на индексы $\alpha, \beta=1,2, \ldots, 9$ так, что $(1,1) \mapsto 1,(1,2) \mapsto 2, \ldots,(3,3) \mapsto 9$, получим систему линейных алгебраических уравнений $F_{\alpha}^{\beta} Q_{\alpha}=0$. Условием существования 
нетривиального решения $Q_{\alpha}(\mathbf{x}) \neq 0$ этой системы является $\operatorname{det}\left|F_{\alpha}^{\beta}\right|=0$. Из явного вида $F_{\alpha}^{\beta}$, заданного в (4.8), следует, что это соотношение выполняется при любых направлениях вектора а. В этом легко убедиться, если выписать детерминант в системе координат, где этот вектор имеет компоненты $(0,0,1)$. Решение уравнения (4.8) $Q_{i k}(\mathbf{a})=Q\left(a_{i} a_{k}-\delta_{i k} / 3\right)$ и вид генератора ненарушенной симметрии (4.7) отвечают случаю одноосного нематика. Рассуждая аналогично, нетрудно показать, что функция $f_{u v}$, входящая в выражение для источника $\widehat{F}$, имеет вид $f_{u v}=a_{u} a_{v}-\delta_{u v} / 3$. Следовательно, рассматриваемое жидкокристаллическое состояние описывается статистическим оператором $\widehat{w}=\widehat{w}(Y, \mathbf{a})$, который зависит от термодинамических сил и вектора пространственной анизотропии (директора), причем зависимость равновесных средних от последнего аргумента сохраняется и после термодинамического предельного перехода.

Холестерики. Исследуем случай, когда пространственная симметрия состояния равновесия имеет более сложный характер и определяется равенством

$$
\widehat{P}_{k}(t) \equiv \widehat{P}_{k}-t_{k j} \hat{L}_{j}
$$

где $\widehat{P}_{k}(t)$ - генератор пространственной симметрии, $t_{i k}$ - числовая матрица с комплексными элементами, характеризующая пространственную симметрию. Генератор ненарушенной симметрии для таких неоднородных состояний представляет собой линейную комбинацию операторов импульса и орбитального момента:

$$
\widehat{T}(\mathbf{a}, \mathbf{d}) \equiv a_{i} \hat{L}_{i}+d_{i} \widehat{P}_{i}
$$

где $\mathbf{a}, \mathbf{d}$ - действительные векторные параметры, причем $a^{2}=1$. Условие пространственной симметрии состояния равновесия

$$
\left[\widehat{w}\left(Y^{\prime}\right), \widehat{P}_{k}\right]=0
$$

таких жидких кристаллов следует дополнить условием ненарушенной симметрии состояния равновесия (4.6), где теперь генератор $\widehat{T}$ определяется равенством (4.10). В соответствии с этими условиями симметрии запишем равенства

$$
\mathrm{i} \operatorname{Sp}[\widehat{w}, \widehat{T}(\mathbf{a}, \mathbf{d})] \widehat{Q}_{u v}(\mathbf{x})=0, \quad \mathrm{i} \operatorname{Sp}\left[\widehat{w}, \widehat{P}_{i}(t)\right] \widehat{Q}_{u v}(\mathbf{x})=0 .
$$

Условие (4.11), вид генератора $\widehat{P}_{k}(4.9)$ и алгебра (4.2) приводят к системе уравнений

$$
\nabla_{k} Q_{u v}(\mathbf{x})=t_{k j} Q_{u v}^{j}(\mathbf{x}), \quad t_{k j} \varepsilon_{j s q} t_{q p} Q_{u v}^{p}(\mathbf{x})=0
$$

где введено обозначение $Q_{u v}^{j}(\mathbf{x}) \equiv \varepsilon_{j u \lambda} Q_{\lambda v}(\mathbf{x})+\varepsilon_{j v \lambda} Q_{\lambda u}(\mathbf{x})$. Второе соотношение в (4.13) вытекает из требования отсутствия линейного по координате слагаемого в соотношении пространственной симметрии (4.12) и первого соотношения в (4.12). Условие ненарушенной симметрии в (4.12) с учетом вида генератора (4.10) приводит к уравнениям

$$
a_{i} \varepsilon_{i k l} t_{l j} Q_{u v}^{j}(\mathbf{x})=0, \quad \underline{a}_{i} Q_{u v}^{i}(\mathbf{x})=0
$$


где $\underline{a}_{i} \equiv a_{i}+d_{j} t_{j i}$. Дополнительные связи параметров генераторов симметрии, введенных соотношениями (4.9), (4.10), установим на основании тождества Якоби. Для операторов $\widehat{w}, \widehat{T}, \widehat{\mathbf{P}}$, принимая во внимание свойства симметрии (4.6), (4.11), приходим к равенству $\operatorname{Sp}\left[\widehat{w},\left[\widehat{T}, \widehat{P}_{k}\right]\right] \widehat{Q}_{u v}(\mathbf{x})=0$. Откуда, учитывая $(4.2),(4.9),(4.10)$, получаем соотношения

$$
a_{i} t_{k j} \varepsilon_{i j \lambda} \varepsilon_{\lambda s l} t_{l p} Q_{u v}^{p}(\mathbf{x})=0, \quad a_{i} t_{k j} \varepsilon_{i j p} Q_{u v}^{p}(\mathbf{x})=0 .
$$

Используя тождество Якоби для операторов $\widehat{w}, \widehat{P}_{i}, \widehat{P}_{k}$ и учитывая свойство пространственной симметрии (4.11), приходим к равенству $\operatorname{Sp}\left(\left[\widehat{w},\left[\widehat{P}_{i}, \widehat{P}_{k}\right]\right] \widehat{Q}_{u v}(\mathbf{x})\right)=0$. Отсюда следуют уравнения

$$
t_{i \lambda} t_{k j} \varepsilon_{\lambda j q} \varepsilon_{q s l} t_{l \sigma} Q_{u v}^{\sigma}(\mathbf{x})=0, \quad t_{i \lambda} t_{k j} \varepsilon_{\lambda j q} Q_{u v}^{q}(\mathbf{x})=0 .
$$

Система уравнений (4.13)-(4.16) полностью определяет допустимую структуру параметров генератора симметрии и вид параметра порядка в изучаемом неоднородном состоянии равновесия. Покажем, что решение этой системы уравнений может быть представлено в виде

$$
Q_{i k}(\mathbf{x})=Q(Y)\left\{\left(m_{i} \cos \varphi(\mathbf{x})+n_{i} \sin \varphi(\mathbf{x})\right)\left(m_{k} \cos \varphi(\mathbf{x})+n_{k} \sin \varphi(\mathbf{x})\right)-\frac{\delta_{i k}}{3}\right\} .
$$

Действительно, если подставить это выражение для параметра порядка в уравнения (4.13), то уравнения выполняются тождественно. Далее, первое соотношение в (4.14) приводит при а $\| \mathbf{l}$ к равенству $t=-l / \mathbf{d} \mathbf{l}$. Матрица $t_{i k}$, задающая генератор пространственной симметрии (4.9), при этом имеет вид $t_{i k}=t l_{i} l_{k}$. Аналогично можно показать, что и остальные уравнения в (4.15), (4.16) справедливы при этих же условиях на параметры генераторов симметрии. В рассматриваемом случае неоднородная структура параметра порядка описывает холестерическое состояние и характеризуется функцией $\varphi(\mathbf{x})=\varphi-\mathbf{t x}$, где вектор холестерической спирали $\mathbf{t}$ направлен по оси $\mathbf{l}=\mathbf{m} \times \mathbf{n}$, величина $2 \pi / t$ имеет физический смысл шага этой спирали. Решение подобного вида для параметра порядка ранее было получено в работе [17] из условия минимума модельного выражения энергии как функции поля директора. Наличие двух векторов в генераторе пространственной симметрии приводит к возможности существования прямой и скошенной холестерических спиралей.

Двухосные нематики. Рассмотрим теперь однородные состояния жидких кристаллов, генератор ненарушенной симметрии которых имеет вид

$$
\widehat{T}(\mathbf{a}, \mathbf{m}, \mathbf{n}) \equiv a_{i} \hat{L}_{i}(\mathbf{m}, \mathbf{n}) .
$$

Этот вид генератора, как мы увидим ниже, соответствует двухосным жидкокристаллическим состояниям. Здесь обобщенный оператор орбитального момента определяется равенствами

$$
\hat{L}_{i}(\mathbf{m}, \mathbf{n}) \equiv \hat{L}_{i}+\hat{L}_{i}^{\mathbf{m}}+\hat{L}_{i}^{\mathbf{n}}, \quad \hat{L}_{i}^{\mathbf{m}} \equiv-\mathrm{i} \varepsilon_{i k l} m_{k} \frac{\partial}{\partial m_{l}}, \quad \hat{L}_{i}^{\mathbf{n}} \equiv-\mathrm{i} \varepsilon_{i k l} n_{k} \frac{\partial}{\partial m_{l}}
$$


(векторы $\mathbf{n}$ и $\mathbf{m}$ единичны и ортогональны, $\mathbf{n m}=0$ ) и имеет смысл генератора двухосной пространственной симметрии. В силу определения (4.19) справедливо соотношение i $\left[\hat{L}_{i}(\mathbf{m}, \mathbf{n}), \hat{L}_{k}(\mathbf{m}, \mathbf{n})\right]=-\varepsilon_{i k l} \hat{L}_{l}(\mathbf{m}, \mathbf{n})$. Используя условие симметрии (4.6) при $Y_{i}=0$ с генератором, заданным соотношениями (4.18), (4.19), приходим к уравнению

$$
a_{i}\left(\varepsilon_{i u j} Q_{j v}+\varepsilon_{i v j} Q_{j u}+\varepsilon_{i k l} m_{k} \frac{\partial Q_{u v}}{\partial m_{l}}+\varepsilon_{i k l} n_{k} \frac{\partial Q_{u v}}{\partial n_{l}}\right)=0 .
$$

Решение этого уравнения ищем в виде $Q_{u v} \equiv Q_{1}\left(e_{u}^{(1)} e_{v}^{(1)}-\delta_{u v} / 3\right)+Q_{2}\left(e_{u}^{(2)} e_{v}^{(2)}-\delta_{u v} / 3\right)$, где единичные векторы $\mathbf{e}^{(1)}$ и $\mathbf{e}^{(2)}$ определены равенствами $\mathbf{e}^{(1)} \equiv \mathbf{m} \cos \varphi+\mathbf{n} \sin \varphi$, $\mathbf{e}^{(2)} \equiv-\mathbf{m} \sin \varphi+\mathbf{n} \cos \varphi$. Учитывая далее формулы

$$
\frac{\partial n_{i}}{\partial n_{k}}=\frac{\partial m_{i}}{\partial m_{k}} \equiv \delta_{i k}-n_{i} n_{k}-m_{i} m_{k}=l_{i} l_{k},
$$

преобразуем уравнение (4.20) к следующему виду:

$$
\begin{gathered}
a_{i} \varepsilon_{i u j}\left(Q_{1} e_{j}^{(1)} e_{v}^{(1)}+Q_{2} e_{j}^{(1)} e_{v}^{(1)}\right)+a_{i} \varepsilon_{i v j}\left(Q_{1} e_{j}^{(1)} e_{u}^{(1)}-+Q_{2} e_{j}^{(1)} e_{u}^{(1)}\right)= \\
=Q_{1}(\operatorname{an} \cos \varphi-\operatorname{am} \sin \varphi)\left(l_{u} e_{v}^{(1)}+l_{v} e_{u}^{(1)}\right)- \\
\quad-Q_{2}(\operatorname{an} \sin \varphi+\operatorname{am} \cos \varphi)\left(l_{u} e_{v}^{(2)}+l_{v} e_{u}^{(2)}\right)
\end{gathered}
$$

Для определения допустимых значений вектора а ищем его в виде разложения по ортонормированному реперу: $a_{i}=\alpha n_{i}+\beta m_{i}+\gamma l_{i}$, где числа $\alpha, \beta, \gamma$ связаны соотношением $\alpha^{2}+\beta^{2}+\gamma^{2}=1$. В результате получим условие $\gamma\left(Q_{1}-Q_{2}\right)\left(e_{u}^{(1)} e_{v}^{(2)}+e_{v}^{(1)} e_{u}^{(2)}\right)=0$. Отсюда следует, что при $Q_{1} \neq 0, Q_{2} \neq 0, Q_{1} \neq Q_{2}$ параметр $\gamma=0$. Это решение описывает пространственно-однородный двухосный нематик:

$$
Q_{u v}=Q_{1}\left(e_{u}^{(1)} e_{v}^{(1)}-\frac{\delta_{u v}}{3}\right)+Q_{2}\left(e_{u}^{(2)} e_{v}^{(2)}-\frac{\delta_{u v}}{3}\right)
$$

Другое решение получается, если $Q_{1}=Q_{2}$. В этом случае вектор а произволен. В силу соотношения $m_{u} m_{v}+n_{u} n_{v}+l_{u} l_{v}=\delta_{u v}$ параметр порядка приобретает вид

\begin{tabular}{|c|c|c|}
\hline $\begin{array}{c}\text { Состояние } \\
\text { равновесия }\end{array}$ & $\begin{array}{c}\text { Генератор } \\
\text { ненарушенной } \\
\text { симметрии }\end{array}$ & Параметр порядка \\
\hline $\begin{array}{c}\text { Одноосный } \\
\text { нематик }\end{array}$ & $a_{i} \hat{L}_{j}$ & $Q_{i k}(\mathbf{a})=Q\left(a_{i} a_{k}-\delta_{i k} / 3\right)$ \\
\hline $\begin{array}{l}\text { Двухосный } \\
\text { нематик }\end{array}$ & $\begin{array}{c}\left(\alpha n_{i}+\beta m_{i}\right) \hat{L}_{i}(\mathbf{m}, \mathbf{n}) \\
\alpha^{2}+\beta^{2}=1\end{array}$ & $\begin{array}{c}Q_{1}\left(e_{i}^{(1)} e_{k}^{(1)}-\delta_{i k} / 3\right)+Q_{2}\left(e_{i}^{(2)} e_{k}^{(2)}-\delta_{i k} / 3\right), \\
e_{i}^{(1)}=m_{i} \cos \varphi+n_{i} \sin \varphi, e_{i}^{(2)}=-m_{i} \sin \varphi+n_{i} \cos \varphi\end{array}$ \\
\hline
\end{tabular}
$Q_{u v}=-Q\left(l_{u} l_{v}-\delta_{u v} / 3\right)$ и соответствует одноосному нематику. Полученные результаты классификации однородных состояний равновесия жидких кристаллов приведены в табл. 1 .

ТАБлицА 1. Классификация однородных состояний равновесия жидких кристаллов. 
Двойная спираль. Рассматриваемый случай жидкокристаллического упорядочения может быть охарактеризован генератором ненарушенной симметрии вида

$$
\widehat{T}(\mathbf{a}, \mathbf{d}, \mathbf{m}, \mathbf{n}) \equiv a_{i} \hat{L}_{i}(\mathbf{m}, \mathbf{n})+d_{i} \widehat{P}_{i}(t, \mathbf{m}, \mathbf{n}) .
$$

Здесь генератор обобщенного орбитального момента задан равенством (4.19), а генератор пространственной симметрии определяется соотношением

$$
\widehat{P}_{k}(t, \mathbf{m}, \mathbf{n}) \equiv \widehat{P}_{k}-t_{k j} \hat{L}_{j}(\mathbf{m}, \mathbf{n}) .
$$

Допустимые ограничения на параметры генераторов ненарушенной и пространственной симметрий находятся из соотношений

$$
\mathrm{iSp}[\widehat{w}, \widehat{T}(\mathbf{a}, \mathbf{d}, \mathbf{m}, \mathbf{n})] \widehat{Q}_{u v}(\mathbf{x})=0, \quad \mathrm{i} \operatorname{Sp}\left[\widehat{w}, \widehat{P}_{i}(t, \mathbf{m}, \mathbf{n})\right] \widehat{Q}_{u v}(\mathbf{x})=0
$$

Дополнительные соотношения возникают при учете тождества Якоби для операторов $\widehat{w}, \widehat{P}_{i}, \widehat{P}_{k}$ и $\widehat{w}, \widehat{T}, \widehat{P}_{k}$ :

$$
\operatorname{Sp}\left[\widehat{w},\left[\widehat{T}, \widehat{P}_{k}\right]\right] \widehat{Q}_{u v}(\mathbf{x})=0, \quad \operatorname{Sp}\left[\widehat{w},\left[\widehat{P}_{i}, \widehat{P}_{k}\right]\right] \widehat{Q}_{u v}(\mathbf{x})=0 .
$$

Из соотношений (4.25), учитывая алгебру (4.2), получим уравнения,

$$
\begin{gathered}
\nabla_{k} Q_{u v}(\mathbf{x})=t_{k j} Q_{u v}^{j}(\mathbf{x}), \quad t_{k j} \varepsilon_{j s q} t_{q p} Q_{u v}^{p}(\mathbf{x})=0 \\
a_{i} \varepsilon_{i k l} t_{l j} Q_{u v}^{j}(\mathbf{x})=0, \quad \underline{a}_{i} Q_{u v}^{j}(\mathbf{x})=0
\end{gathered}
$$

где величина $Q_{u v}^{j}(\mathbf{x})$ задается равенством

$$
Q_{u v}^{j}(\mathbf{x}) \equiv \varepsilon_{j u \lambda} Q_{\lambda u}(\mathbf{x})+\varepsilon_{j v \lambda} Q_{\lambda u}(\mathbf{x})+\varepsilon_{j s l} n_{s} \frac{\partial Q_{u v}(\mathbf{x})}{\partial n_{l}}+\varepsilon_{j s l} m_{s} \frac{\partial Q_{u v}(\mathbf{x})}{\partial m_{l}} .
$$

Из тождеств Якоби получаем уравнения

$$
\begin{aligned}
a_{i} t_{k j} \varepsilon_{i j \lambda} \varepsilon_{\lambda s l} t_{l p} Q_{u v}^{p}(\mathbf{x}) & =0, \quad a_{i} t_{k j} \varepsilon_{i j p} Q_{u v}^{p}(\mathbf{x})=0, \\
t_{i \lambda} t_{k j} \varepsilon_{\lambda j q} \varepsilon_{q s l} t_{l \sigma} Q_{u v}^{\sigma}(\mathbf{x}) & =0, \quad t_{i \lambda} t_{k j} \varepsilon_{\lambda j q} Q_{u v}^{q}(\mathbf{x})=0 .
\end{aligned}
$$

Поступая далее так же, как и при рассмотрении одноосного холестерика, нетрудно показать, что при $\mathbf{a} \| \mathbf{l}$ и $t=-l / \mathbf{d l}$ уравнения (4.27)-(4.29) выполняются, а параметр порядка в состоянии равновесия приобретает вид

$$
Q_{i k}(\mathbf{x})=Q_{1}\left(e_{i}^{(1)}(\mathbf{x}) e_{k}^{(1)}(\mathbf{x})-\frac{\delta_{i k}}{3}\right)+Q_{2}\left(e_{i}^{(2)}(\mathbf{x}) e_{k}^{(2)}(\mathbf{x})-\frac{\delta_{i k}}{3}\right),
$$

где $e_{i}^{(1)}(\mathbf{x})=m_{i} \cos \varphi(\mathbf{x})+n_{i} \sin \varphi(\mathbf{x}), e_{i}^{(2)}(\mathbf{x})=-m_{i} \sin \varphi(\mathbf{x})+n_{i} \cos \varphi(\mathbf{x})-$ единичные и взаимно ортогональные векторы, а зависимость фазы от координаты имеет вид $\varphi(\mathbf{x})=\varphi-\mathbf{l} \mathbf{x} / \mathbf{d} \mathbf{l}$. Заметим, что решение с пространственной структурой двойной спирали напоминает структуру молекулы ДНК [18]. Комплементарные пары Уотсона-Крика аденин-тимин и гуанин-цитозин лежат в плоскости векторов $\mathbf{e}^{(1)}(\mathbf{x}), \mathbf{e}^{(2)}(\mathbf{x})$ и поворачиваются вокруг оси анизотропии $\mathbf{l}$ при смещении координаты. Случай коллинеарных векторов $\mathbf{l}$ и $\mathbf{d}$ отвечает В-форме ДНК. При этом 
ТАБлицА 2. Классификация неоднородных состояний равновесия жидких кристаллов.

\begin{tabular}{|c|c|c|c|}
\hline $\begin{array}{c}\text { Состояние } \\
\text { равновесия }\end{array}$ & $\begin{array}{c}\text { Генератор } \\
\text { ненарушенной } \\
\text { симметрии }\end{array}$ & $\begin{array}{c}\text { Генератор } \\
\text { пространственной } \\
\text { симметрии }\end{array}$ & $\begin{array}{c}\text { Параметр порядка } \\
\text { холестерик }\end{array}$ \\
\hline$l_{i} \hat{L}_{i}+d_{i} \widehat{P}_{i}$ & $\widehat{P}_{k}+\frac{l_{k}}{\mathbf{d} \mathbf{l}} l_{i} \hat{L}_{i}$ & $\begin{array}{c}Q\left(e_{i}^{(1)}(\mathbf{x}) e_{k}^{(1)}(\mathbf{x})-\delta_{i k} / 3\right), \\
e_{i}^{(1)}(x)=m_{i} \cos \varphi(\mathbf{x})+n_{i} \sin \varphi(\mathbf{x}), \\
\varphi(\mathbf{x})=\varphi-\mathbf{l x} / \mathbf{d} \mathbf{l}\end{array}$ \\
\hline двойная & $l_{i} \hat{L}_{i}(\mathbf{m}, \mathbf{n})+d_{i} \widehat{P}_{i}$, & $\widehat{P}_{k}+\frac{l_{k}}{\mathbf{d} \mathbf{l}} l_{i} \hat{L}_{i}(\mathbf{m}, \mathbf{n})$ & $\begin{array}{c}Q_{1}\left(e_{i}^{(1)}(\mathbf{x}) e_{k}^{(1)}(\mathbf{x})-\delta_{i k} / 3\right)+ \\
+Q_{2}\left(e_{i}^{(2)}(\mathbf{x}) e_{k}^{(2)}(\mathbf{x})-\delta_{i k} / 3\right), \\
\text { спираль }\end{array}$
\end{tabular}

комплементарные пары располагаются в плоскости, ортогональной оси спирали. Если векторы $\mathbf{l}$ и $\mathbf{d}$ неколлинеарны, то такая пространственная структура представляет собой А-форму, и имеет место упорядочение пространственной структуры типа скошенной спирали. Плоскости комплементарных пар в этом случае располагаются под углом к оси спирали. Различие в знаке скалярного произведения векторов $(\mathbf{l d}>0$ или $\mathbf{l d}<0)$ означает возможность физической реализации правосторонней и левосторонней спиралей.

А-смектик. Для этого состояния равновесия жидких кристаллов условия пространственной симметрии имеют вид

$$
\left[\widehat{w}, l_{i} \hat{L}_{i}\right]=0, \quad \varepsilon_{i k l} l_{k}\left[\widehat{w}, \widehat{P}_{l}\right]=0, \quad e^{\mathrm{i} \widehat{P}_{k} l_{k} b} \widehat{w} e^{-\mathrm{i} \widehat{P}_{k} l_{k} b}=\widehat{w} .
$$

Они показывают, что среда обладает одноосной пространственной анизотропией. Вдоль оси анизотропии 1 имеет место периодическая кристаллическая структура (одномерный кристалл), а в плоскости, ортогональной этой оси, реализуется двумерное однородное и изотропное состояние конденсированной среды (жидкая фаза). Первое соотношение в (4.30) и операторная алгебра (4.2) приводят к равновесной структуре параметра порядка:

$$
Q_{u v}(\mathbf{x}, Y, \mathbf{l}, b)=Q(\mathbf{x}, Y, \mathbf{l}, b)\left(l_{u} l_{v}-\frac{1}{3} \delta_{u v}\right) .
$$

В рассматриваемом случае модуль параметра порядка зависит от пространственной координаты. Эту зависимость мы будем искать в виде

$$
Q(\mathbf{x}, Y, \mathbf{l}, b)=Q(\varphi(\mathbf{x}, \mathbf{l}, b, Y)), \quad \varphi(\mathbf{x}, \mathbf{l}, b)=\varphi(\mathbf{x}+k \mathbf{l} b) .
$$

Второе условие в (4.30) приводит к равенству $[\mathbf{l} \times \nabla \varphi]=0$, откуда находим $\varphi(\mathbf{x})=$ $\varphi(0)+p \mathbf{l x}$. Так как из последнего соотношения в (4.30) следует периодичность 
фазовой функции, $\varphi(\mathbf{x})=\varphi(\mathbf{x}+b \mathbf{l})$, из ее разложения в ряд Фурье

$$
\varphi(\mathbf{x})=\sum_{k} \varphi_{k} e^{\mathrm{i} k p \mathbf{l} \mathbf{x}}=\sum_{k} \varphi_{k} e^{\mathrm{i} k p \mathbf{l}(\mathbf{x}+b \mathbf{l})}
$$

$(k-$ натуральное число) находим значения параметра $p=2 \pi / b$. Таким образом, пространственная зависимость функции $\varphi(\mathbf{x})$ имеет вид $\varphi(\mathbf{x})=\sum_{k} \varphi_{k} e^{2 \pi \mathrm{i} k \mathbf{l} \mathbf{x} / b}$.

C-смектик. Условия пространственной симметрии этого типа жидких кристаллов имеют вид

$$
\left[\widehat{w}, m_{i} \hat{L}_{i}\right]=0, \quad \varepsilon_{i k l} l_{k}\left[\widehat{w}, \widehat{P}_{l}\right]=0, \quad e^{\mathrm{i} \widehat{P}_{k} l_{k} b} \widehat{w} e^{-\mathrm{i} \widehat{P}_{k} l_{k} b}=\widehat{w}
$$

где векторы $\mathbf{l}$ и $\mathbf{m}$ удовлетворяют соотношениям $l^{2}=m^{2}=1, \mathbf{l m}=\cos \theta$. В каждой плоскости длинные оси молекул (оптическая ось $\mathbf{m}$ ) наклонены по отношению к кристаллической оси $\mathbf{l}$ на угол $\theta$. Условия симметрии (4.33) приводят к равновесной структуре параметра порядка:

$$
Q_{u v}(\mathbf{x}, Y, \mathbf{l}, \mathbf{m})+Q_{u v}(\varphi(\mathbf{x}), Y)\left(m_{u} m_{v}-\frac{1}{3} \delta_{u v}\right), \quad \varphi(\mathbf{x})=\sum_{k} \varphi_{k} e^{2 \pi \mathrm{i} k \mathbf{l} \mathbf{x} / b} .
$$

Аналогичным образом могут быть рассмотрены дискотические жидкие кристаллы.

\section{5. ВЫВОды}

Предложенный статистический подход к классификации состояний равновесия конденсированных сред с нарушенной симметрией не содержит каких-либо модельных предположений о виде свободной энергии и не использует требования близости температуры к точке фазового перехода. Метод классификации вырожденных состояний конденсированных сред применен при описании квадрупольных магнетиков с векторным и квадрупольным параметрами порядка [19], [20]. Получено аналитическое решение задачи классификации состояний равновесия для сверхтекучих систем: сверхтекучего ${ }^{3} \mathrm{He}$ [5], сверхтекучей среды с d-спариванием [21] и раствора ферми-жидкостей с векторным параметром порядка [22].

Благодарности. Автор выражает благодарность РФФИ за финансовую поддержку (грант № 09-01-00086_а).

\section{Список литературы}

[1] N. Bogolubov, Physica, 26, Suppl. 1 (1960), s1-s16.

[2] Н. Н. Боголюбов, Н. Н. Боголюбов (мл.), Введение в квантовую статистическую механику, Наука, М., 1984.

[3] А.И. Ахиезер, С. В. Пелетминский, Методъ статистической физики, Наука, М., 1977.

[4] Н. Н. Боголюбов (мл.), М. Ю. Ковалевский, А. М. Курбатов, С. В. Пелетминский, А. Н. Тарасов, УФН, 159:4 (1989), 585-620.

[5] М.Ю.Ковалевский, С.В.Пелетминский, ЭЧАЯ, 33:6 (2002), 1357-1445. 
[6] М.Ю.Ковалевский, С.В. Пелетминский, Статистическая механика квантовых жидкостей и кристаллов, Физматлит, М., 2006.

[7] Л. Д. Ландау, ЖЭЭТФ, 7 (1937), 18-33; "К теории фазовых переходов. I", Собрание трудов, Т. 1, ред. Б. М. Лифшиц, Наука, М, 1969, 234-252.

[8] G. Barton, M. A. Moore, J. Phys. C, 7:17 (1974), 2989-3000.

[9] P. G. de Gennes, J. Prost, The Physics of Liquid Crystals, Oxford Univ. Press, Oxford, 1995.

[10] М. Клеман, О.Д. Лаврентович, Основы физики частично упорядоченных сред, Физматлит, М., 2007.

[11] D. Belitz, T. R. Kirkpatrick, T. Vojta, Rev. Modern Phys., 77:2 (2005), 579-632.

[12] V. P. Mineev, Sov. Sci. Rev. A, 2 (1980), 173-246; Topologically Stable Defects and Solitons in Ordered Media, Classic Rev. Phys., 1, Harwood Academic, Amsterdam, 1998.

[13] F. W. Nijhoff, H. W. Capel, A. Breems, Phys. A, 130:3 (1985), 375-411.

[14] A. M. J. Schakel, F. A. Bais, J. Phys., 1:9 (1989), 1743-1752.

[15] H. W. Capel, "Group theory and phases of superfluid ${ }^{3} \mathrm{He}$ ", Selected Topics in Statistical Mechanics, eds. A. A. Logunov, N. N. Bogolubov, Jr., V. G. Kadyshevsky A. S. Shumovsky, World Sci., Teaneck, NJ, 1990, 73-83.

[16] D. Vollhardt, P. Wölfle, The Superfluid Phases of Helium 3, Taylor \& Francis, New York, 1990.

[17] Е. Кац, В. В. Лебедев, Динамика жидких кристаллов, УРСС, М., 1988.

[18] А. В. Финкельштейн, О. Б. Птицин, Физика белка, Курс лекций, Книжный Дом "Университет", М., 2002.

[19] Н. Н. Боголюбов (мл.), М. Ю. Ковалевский, УФЖ, 50:8А (2005), А104-А112.

[20] Д. А. Демьяненко, М. Ю. Ковалевский, ФНТ, 33:11 (2007), 1271-1281.

[21] А. П. Ивашин, М. Ю. Ковалевский, Н. Н. Чеканова, ФНТ, 30:9 (2004), 920-927.

[22] M. Y. Kovalevskii, A. A. Rozhkov, L. V. Logvinova, Phys. A, 336:3-4 (2004), 271-293. 